\title{
Erratum: All Entangled States Can Demonstrate Nonclassical Teleportation [Phys. Rev. Lett. 119, 110501 (2017)]
}

Daniel Cavalcanti, Paul Skrzypczyk, and Ivan Šupić

(Received 11 October 2017; published 10 November 2017)

DOI: 10.1103/PhysRevLett.119.199902

In the published version we claimed that $\mathcal{T}_{R}=E_{R}$ for a partial Bell state measurement (BSM). This is incorrect. In fact this equality holds in the case of a full BSM, while $\mathcal{T}_{R} \propto E_{R}$ for a partial BSM. Full details can be found in the updated Supplemental Material. The main result that all entangled states lead to nonclassical teleportation is unaffected. 